\title{
Identifying Gamma-Ray Burst Remnants through Positron Annihilation Radiation
}

\section{Citation}

Furlanetto, Steven R., and Abraham Loeb. 2002. “Identifying Gamma-Ray Burst Remnants through Positron Annihilation Radiation." The Astrophysical Journal 569 (2): L91-94. https:// doi.org/10.1086/340772.

\section{Permanent link}

http://nrs.harvard.edu/urn-3:HUL.InstRepos:41393322

\section{Terms of Use}

This article was downloaded from Harvard University's DASH repository, and is made available under the terms and conditions applicable to Other Posted Material, as set forth at http:// nrs.harvard.edu/urn-3:HUL.InstRepos:dash.current.terms-of-use\#LAA

\section{Share Your Story}

The Harvard community has made this article openly available.

Please share how this access benefits you. Submit a story.

Accessibility 
The Astrophysical Journal, 569:L91-L94, 2002 April 20

(c) 2002. The American Astronomical Society. All rights reserved. Printed in U.S.A.

\title{
IDENTIFYING GAMMA-RAY BURST REMNANTS THROUGH POSITRON ANNIHILATION RADIATION
}

\author{
Steven R. Furlanetto and Abraham Loeb \\ Harvard-Smithsonian Center for Astrophysics, 60 Garden Street, Cambridge, MA 02138; sfurlanetto@cfa.harvard.edu, aloeb@cfa.harvard.edu \\ Received 2002 March 4; accepted 2002 March 19; published 2002 March 26
}

\begin{abstract}
We model the annihilation of relic positrons produced in a gamma-ray burst (GRB) after its afterglow has faded. We find that the annihilation signal from at least one GRB remnant in the Milky Way should be observable with future space missions such as the International Gamma-Ray Astrophysical Laboratory and the Energetic $X$-ray Imaging Survey Telescope, provided that the gas surrounding the GRB source has the typical density of the interstellar medium, $\lesssim 1 \mathrm{~cm}^{-3}$. Three fortunate circumstances conspire to make the signal observable. First, unlike positrons in a standard supernova, the GRB positrons initially travel at a relativistic speed and remain ahead of any nonrelativistic ejecta until the ejecta become rarefied and the annihilation time becomes long. Second, the GRB remnant remains sufficiently hot $\left(T \gtrsim 5 \times 10^{5} \mathrm{~K}\right)$ for a strong annihilation line to form without significant smearing by three-photon decay of positronium. Third, the annihilation signal persists over a time longer than the average period between GRB events in the Milky Way.
\end{abstract}

Subject headings: gamma rays: bursts - supernova remnants - X-rays: ISM

\section{INTRODUCTION}

The detailed nature of the central engine of gamma-ray bursts (GRBs) is still unknown (van Paradijs, Kouveliotou, \& Wijers 2000; Piran 2000 and references therein). Attempts to derive empirical constraints on the environment (Djorgovski et al. 2001 and references therein), the frequency (Schmidt 2001), the collimation angles and energy output (Frail et al. 2001; Freedman \& Waxman 2001; Panaitescu \& Kumar 2001b; Piran et al. 2001), or the possible association of GRBs with supernovae (SNe; Bloom et al. 1999; Kulkarni et al. 2000; Reichart 2001) are compromised by the difficulty of observing GRBs across large cosmological distances. Obviously, identification of old GRB remnants in the local universe would provide much better insight into the nature of GRB progenitors (Loeb \& Perna 1998; Woods \& Loeb 1999; Perna, Raymond, \& Loeb 2000; Paczyński 2001; Ayal \& Piran 2001).

Given the recently inferred similarity between the energy output in SNe and GRBs, $\sim 10^{51}$ ergs (Frail et al. 2001), it now appears difficult to separate the late evolution stages of their remnants hydrodynamically. Even if a GRB explosion is initially highly beamed, the asymmetry of the blast wave it generates in the surrounding interstellar medium (ISM) would be erased on an isotropization timescale $t_{\text {iso }} \sim 6 \times 10^{3}\left(E_{51} / n_{0}\right)^{1 / 3} \mathrm{yr}$ (Ayal \& Piran 2001), where $E_{51}$ is the kinetic energy output of the GRB in units of $10^{51} \mathrm{ergs}$ and $n_{0}=\rho_{\mathrm{ISM}} / \mu_{b} m_{p}$ is the ambient number density of atoms in units of $\mathrm{cm}^{-3}$ for a mean molecular weight $\mu_{b}=1.4$. Subsequently, the blast wave expands just as in a normal SN remnant. Any nonrelativistic, SN-like ejecta would moderate the initial GRB asymmetry as soon as it overtakes the decelerating GRB shock (Piran \& Ayal 2002). External anisotropy may also result from the interaction of the remnant blast wave with a nonuniform ISM. Thus, morphological studies alone cannot unambiguously identify GRB remnants. The alternative method of seeking spectral signatures of the photoionized regions around GRBs (Perna et al. 2000) also suffers from potential confusion with SNe occurring in unusual environments.

In this Letter, we point out that positrons produced during the early relativistic GRB phase offer a powerful tool for identifying GRB remnants. We model the expansion of a GRB remnant and show that a well-defined annihilation line should be visible until radiative cooling becomes important, at which time the remaining positrons annihilate rapidly (see also Dermer \& Böttcher 2000).

\section{MODEL OF GRB REMNANTS}

We model the initial GRB explosion as two highly relativistic jets of material moving in opposite directions, initially covering a fraction $f_{b}$ of a sphere surrounding the GRB. We assume that a fraction $\xi_{+}$of the GRB energy is transformed into $e^{+}$rest mass. The positrons can be produced either during the burst itself (Cavallo \& Rees 1978; Shemi \& Piran 1990) or by interactions of the gamma rays with the ambient medium (Thompson \& Madau 2000; Dermer \& Böttcher 2000; Mészáros, Ramirez-Ruiz, \& Rees 2001). We scale our results to a fiducial value of $\xi_{-2.3}=\left(\xi_{+} / 0.005\right)$ corresponding to half of the GRB energy in positrons with a bulk Lorentz factor $\gamma \sim$ 100 as required by the compactness argument (Piran 2000). We therefore assume a total of $N_{+}=6 \times 10^{54} \xi_{-2.3} E_{51}$ positrons in the GRB.

The positrons initially travel at a relativistic speed just ahead of the GRB jet. The jet begins to decelerate when it reaches a radius $R_{j} \sim 0.2\left(E_{51} / n_{0}\right)^{1 / 3} \mathrm{pc}$ (Rhoads 1997$)$. At this point, it begins to expand sideways and slows exponentially to nonrelativistic speeds. Thereafter, simulations have shown that $R_{j} \propto t^{1 / 3}$ until the remnant isotropizes at $t_{\text {iso }}$ and begins a SedovTaylor spherical expansion (Ayal \& Piran 2001).

If the commonly hypothesized link between GRBs and SNe exists, then the jet will be followed by SN ejecta of mass $M_{\mathrm{ej}} \sim 10 M_{\odot}$. The ejecta expand freely behind the jet and eventually impact the (decelerating) positrons at a time $t_{\text {mix }} \sim$ $110\left(E_{51} / n_{0}\right)^{1 / 3} v_{4}^{-3 / 2} \mathrm{yr}$, where $v_{4}$ is the ejecta velocity in units of $10^{4} \mathrm{~km} \mathrm{~s}^{-1}$ (Piran \& Ayal 2002). At this point, the density is $n_{\text {mix }} \gtrsim 50 M_{1}\left(n_{0} / E_{51}\right) v_{4}^{3 / 2} \mathrm{~cm}^{-3}$, assuming that the ejecta are distributed uniformly throughout the remnant and where $M_{1}=M_{\mathrm{ej}} / 10 M_{\odot}$. If mixing of the ejecta and shocked gas is efficient, then the ejecta-dominated expansion models of Truelove \& McKee (1999) show that by this point the thermal energy imparted to the swept-up material could heat the remnant to a temperature $T_{\text {mix }} \gtrsim 10^{8} \mathrm{~K}\left(E_{51} / M_{1}\right)$. The ratio of the annihilation time to remnant age does not change with time, $\tau_{\text {ann }} / t \sim 500 M_{1}^{-1}\left(E_{51} / n_{0}\right) v_{4}^{-3 / 2}$. Thus, only a small fraction of the 
positrons should annihilate during this very early stage, even if the ejecta are concentrated in a shell of width $\sim 0.1 R_{\text {mix }}$.

This contrasts with the survival of positrons in a standard $\mathrm{SN}$, where a substantial number of positrons can be created through the decay chain ${ }^{56} \mathrm{Ni} \rightarrow{ }^{56} \mathrm{Co} \rightarrow{ }^{56} \mathrm{Fe}+e^{+}$, which has a decay time of $\tau \sim 111$ days and occurs for $18 \%$ of the ${ }^{56} \mathrm{Ni}$ atoms. The SN positrons are produced inside the cool, dense SN ejecta when $\tau_{\text {ann }} / t \lesssim 0.03$ (depending on the uncertain ionization and temperature structure of the ejecta). Therefore, models predict that $\lesssim 10^{52} e^{+}$can escape the SN ejecta (Chan \& Lingenfelter 1993; Milne, The, \& Leising 1999). These models are consistent with the positron production rate inferred from observations of diffuse $e^{+}$annihilation radiation in the Milky Way (Purcell et al. 1997; Kinzer et al. 1996, 2001).

After passing through the external shock, most positrons will rapidly thermalize with the ambient medium while a small fraction $\left(\sim 10^{-3}\right)$ will be reaccelerated by the (collisionless) external shock (Gieseler, Jones, \& Kang 2000). If positrons are produced ahead of the shock, then they will have a characteristic Lorentz factor $\gamma_{+} \sim 30$ (Mészáros et al. 2001). Regardless of their initial state, the positrons cool rapidly through Coulomb collisions and adiabatic decompression as the remnant expands. While they are relativistic, adiabatic cooling leads to energy loss on the dynamical timescale of the remnant, $\tau_{\text {ad }} \sim R_{s} / v_{s}$, where $R_{s}$ and $v_{s}$ are the radius and expansion speed of the (assumed spherical) remnant. Once the positrons become nonrelativistic, their Coulomb cooling time is short, $\tau_{\text {Coul }} \sim 3 \times$ $10^{4}\left(v_{+} / c\right) n_{0}^{-1} \mathrm{yr}$, where $v_{+}$is the positron velocity. Using the analytic expressions for the cooling rates in Furlanetto \& Loeb (2002) supplemented by expansion cooling, we have confirmed that the cooling timescales for $\gamma_{+} \lesssim 100$ are much shorter than the duration of the Sedov phase. We therefore simply assume that all positrons begin in thermal equilibrium with the shocked ambient gas.

Once the remnant isotropizes, it follows the Sedov (1959) solution until radiative cooling becomes important. Although the remnant is initially asymmetric because of the jet geometry, Ayal \& Piran (2001) have shown numerically that even before isotropization, the total volume of the remnant scales with time similarly to a Sedov self-similar blast wave. For this reason, and because $t_{\text {iso }}$ is much smaller than the duration of the adiabatic phase, we treat the remnant boundary as a Sedov blast wave throughout both of these phases. Then $R_{s}=$ $0.314\left(E_{51} / n_{0}\right)^{1 / 5} t_{\mathrm{yr}}^{2 / 5} \mathrm{pc}$, where $t_{\mathrm{yr}}$ is the remnant age in years. The adiabatic index of the ambient medium is taken to be $\Gamma=5 / 3$.

To calculate $N_{+}(t)$, we assume that the positrons are perfectly mixed throughout the interior of the remnant, so that the $e^{+}$ density $n_{+}(r) \propto n_{e}(r)$, where $n_{e}$ is the free electron density. We expect such mixing to occur on a timescale $\sim t_{\text {iso }}$ or faster because of instabilities on the interface between the SN ejecta and the ambient medium (Chevalier, Blondin, \& Emmering 1992; Jun, Jones, \& Norman 1996). The annihilation rate is then

$$
\dot{N}_{+}=-\int_{0}^{R_{s}} n_{e}(r) n_{+}(r) \alpha[T(r)] 4 \pi r^{2} d r
$$

where the radial distributions of $n_{e}$ and $T$ are given by Sedov (1959). Here $\alpha=\alpha_{\mathrm{fa}}+\alpha_{\mathrm{Ps}}$ is the annihilation rate coefficient with contributions from two channels. The first component, $\alpha_{\text {fa }}$, involves direct annihilation with free electrons. In the nonrelativistic regime $\left(T \lesssim 10^{8} \mathrm{~K}\right)$, we use the rate coefficient given by Gould (1989), which includes corrections due to Coulomb focusing. Above this temperature, we use the fitting formula given by Svensson (1982). The second coefficient $\alpha_{\mathrm{Ps}}$ describes radiative recombination to positronium (Ps) and is given by Gould (1989). Although the corresponding expression is strictly valid only in the nonrelativistic regime, Ps formation is subdominant for $T_{\text {ann }}>10^{6} \mathrm{~K}$ and so the errors introduced by applying it to high temperatures are negligible. The Ps lifetime is $\tau_{\mathrm{Ps}}<1.33 \times 10^{-7} \mathrm{~s}$ (depending on the energy level to which recombination occurs), and so we assume that positrons annihilate immediately after forming Ps.

Once the shocked gas cools radiatively to $T \lesssim 10^{4} \mathrm{~K}$, a dense shell begins to form at the shock front. We use the cooling function of Cioffi, McKee, \& Bertschinger (1988), for which the nominal shell formation time is $t_{\mathrm{sf}}=3.61 \times 10^{4} E_{51}^{3 / 14} n_{0}^{-4 / 7} \mathrm{yr}$. The shell formation process is not fully understood, but twodimensional simulations have shown it to be both unstable and violent (Blondin et al. 1998). These simulations also find that the dense shell does not fully form until $\sim 1.5 t_{\mathrm{sf}}$. We therefore show results up to this time with the caveat that once cooling becomes important at $t_{\text {sf }}$ they may no longer be accurate. After shell formation, we expect the positrons to annihilate rapidly. Not only does the density of the shell increase dramatically, but also charge exchange with $\mathrm{H}$ I comes to dominate as the temperature falls below $\sim 10^{5} \mathrm{~K}$ (Bussard, Ramaty, \& Drachman 1979; see § 4). Thus, we do not expect the annihilation signal to persist after the Sedov phase ends.

Even before shell formation, the loss of energy to cooling radiation decelerates the remnant. We use the analytic approximation of Cioffi et al. (1988) to describe the blast-wave size and velocity in this phase (for $t>t_{\mathrm{sf}} / e$, where $e$ is Euler's constant). As there is no analytic understanding of the interior structure of the remnant at this stage, we continue to use profiles from the Sedov (1959) solution, normalized to the total size and velocity of Cioffi et al. (1988). Our results are only weakly dependent on the details of these solutions.

\section{RESULTS}

Figure 1 shows the expected flux of annihilation photons, $F_{\gamma}$, as a function of time for blast waves traveling through media with densities $n_{0}=10^{3}, 10^{2}, 10^{1}, 1$, and $0.1 \mathrm{~cm}^{-3}$ (from top to bottom). For each density, we carry the integration through $1.5 t_{\mathrm{sf}}$. The solid lines show the photon flux from direct annihilation, while the dashed lines show that from Ps formation. We scale our results to $d_{10}$, the remnant distance in units of $10 \mathrm{kpc}$. The photon production rate is nearly proportional to the ambient density because at a fixed temperature $\tau_{\text {ann }} \propto n_{e}^{-1}$. We find that the average temperature of annihilating positrons is $T_{\text {ann }} \approx$ $1.25 T_{s}$, where $T_{s}$ is the postshock temperature. The annihilationweighted density is $n_{\text {ann }} \approx 2.7 n_{0}$. Both of these formulae are valid to within $\sim 5 \%$ for all times and densities. Thus, our model finds that most of the annihilations occur in a shell near the shock front. Throughout most of the remnant lifetime, $5 \times$ $10^{5} \mathrm{~K} \lesssim T_{\text {ann }} \lesssim 10^{7} \mathrm{~K}$. Thus, direct $e^{+} e^{-}$annihilation dominates, with Ps formation becoming important only at late times when the shock velocity has fallen substantially. Note that $F_{\gamma}$ is approximately proportional to $E_{51}$, but the explosion energy also affects $t_{\mathrm{sf}}$.

We next calculate the emission spectrum of the remnant. The spectrum produced by direct annihilation when $k_{\mathrm{B}} T_{e} \ll m_{e} c^{2}$ is given by Svensson, Larsson, \& Poutanen (1996). We ignore Coulomb corrections to the annihilation rate, which is a good assumption for the temperatures of interest here (Gould 1989). 


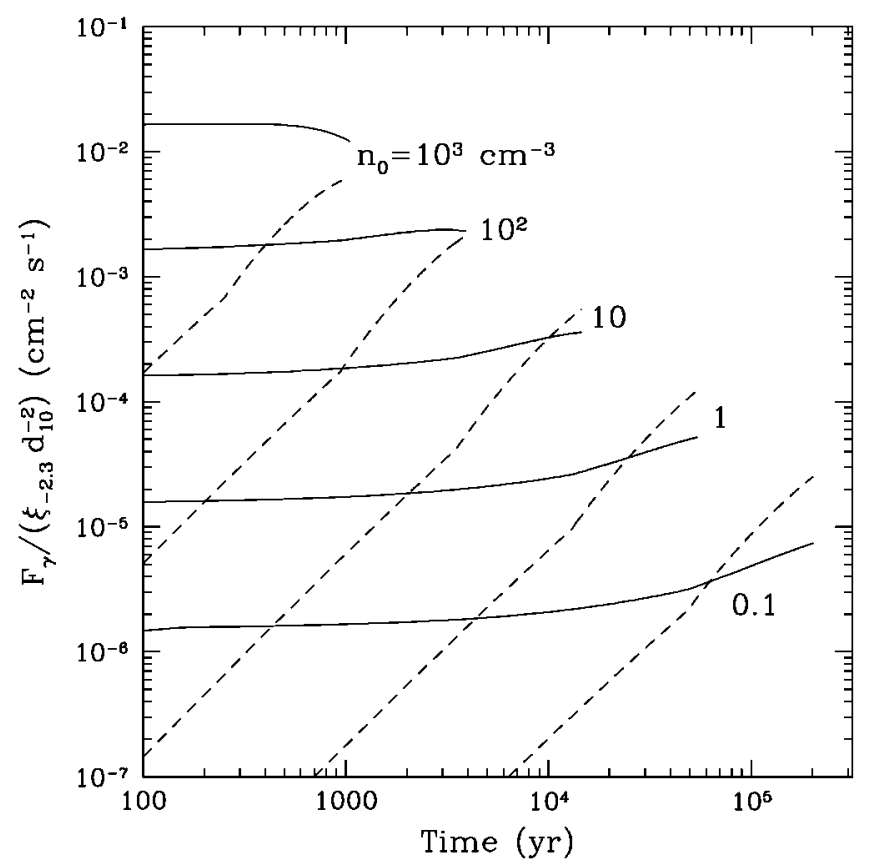

FIG. 1.-Flux of annihilation photons as a function of time for remnants embedded in a medium of density $n_{0}=10^{3}, 10^{2}, 10,1$, and $0.1 \mathrm{~cm}^{-3}$, from top to bottom. Solid curves show the photon flux from free annihilation, while dashed curves show the flux from Ps decay. All results are scaled to a remnant distance $d_{10}=d / 10 \mathrm{kpc}$ and assume $E_{51}=1$.

The decay of Ps is more complicated. One-quarter of the recombinations produce para-Ps, which annihilates into two photons with the spectral distribution given by Gould (1989). Three-quarters of the recombinations produce ortho-Ps, which must decay into three photons in order to conserve angular momentum. The spectrum in this case is a continuum, with a form in the Ps rest frame given by Ore \& Powell (1949). We include the broadening due to the thermal motions of the plasma particles by transforming to the observer frame (Bussard et al. 1979) and using the relativistic Maxwell-Boltzmann velocity distribution. ${ }^{1}$ In calculating the spectra, we assume for simplicity that all annihilations take place at a single temperature $T_{\mathrm{ann}}$. We also neglect the broadening introduced by the expansion speed of the remnant, because the ratio between the thermal $e^{+}$speed and the shock speed is $v_{\mathrm{th}} / v_{s} \sim\left(m_{p} / 2 m_{e}\right)^{1 / 2} \gg 1$, where $m_{e}$ and $m_{p}$ are the electron and proton masses.

Snapshots of the annihilation spectrum at $t / t_{\mathrm{sf}}=0.3,0.6$, $0.9,1.2$, and 1.5 are shown in Figure 2, for media with $n_{0}=1 \mathrm{~cm}^{-3}$ (top panel) and $n_{0}=10^{3} \mathrm{~cm}^{-3}$ (bottom panel). As shown in Figure 1, the number of free annihilations per second varies relatively slowly with remnant age, increasing primarily because of the decreasing $T_{\text {ann }}$. The line also narrows with time: the FWHM is $\sim 30\left(T_{\text {ann }} / 10^{7} \mathrm{~K}\right)^{1 / 2} \mathrm{keV}$. The line is narrower for lower density media at constant $t / t_{\mathrm{sf}}$ because $v_{s}$ and hence $T_{\text {ann }}$ are smaller.

\section{DISCUSSION}

The BATSE data set implies a GRB rate per comoving volume of $\dot{n}_{\text {iso }} \sim 0.5 \mathrm{Gpc}^{-3} \mathrm{yr}^{-1}$, assuming isotropic emission (Schmidt 2001). The actual event rate is estimated to be larger by an average beaming factor, $\left\langle f_{b}^{-1}\right\rangle \sim 520$ (Frail et al. 2001).

\footnotetext{
${ }^{1}$ Note, however, that our expression for $\alpha_{\mathrm{Ps}}$ does not include relativistic corrections, so our continuum level may be an overestimate at very early times when $T_{\text {ann }} \gg 10^{8} \mathrm{~K}$.
}



FIG. 2.- Spectral fluxes as a function of photon energy at various times. Results are shown for $n_{0}=1 \mathrm{~cm}^{-3}$ (top panel) and $n_{0}=10^{3} \mathrm{~cm}^{-3}$ (bottom panel) at times $t / t_{\mathrm{sf}}=0.3$ (dotted curves), 0.6 (dot-dashed curves), 0.9 (shortdashed curves), 1.2 (long-dashed curves), and 1.5 (solid curves). All results are scaled to a remnant distance $d_{10}=d / 10 \mathrm{kpc}$ and assume $E_{51}=1$. The asymmetric line broadening is a property of the annihilation spectrum given by Svensson et al. (1996).

Most models associate GRBs with compact stellar remnants; under the assumption that the GRB event rate is proportional to the star formation rate, we can convert the above rate density to an event rate per unit mass of star formation (e.g., Porciani \& Madau 2001). The expected period between GRB events in the Milky Way is then $\tau_{\mathrm{Mw}} \sim 3 \times$ $10^{4} \operatorname{yr}\left(\dot{n}_{\text {iso }} / 0.5\right)^{-1}\left(\left\langle f_{b}^{-1}\right\rangle / 520\right)^{-1}\left(R_{0} / 0.007\right) R_{\mathrm{MW}}^{-1}$, where $R_{0}$ and $R_{\mathrm{MW}}$ are the present-day average star formation rates in the universe and in the Milky Way in units of $M_{\odot} \mathrm{Mpc}^{-3} \mathrm{yr}^{-1}$ and $M_{\odot} \mathrm{yr}^{-1}$, respectively, and $\dot{n}_{\text {iso }}$ has units of $\mathrm{Gpc}^{-3} \mathrm{yr}^{-1}$.

Together with Figure 1, this suggests that the positron annihilation line should be detectable from at least one Galactic GRB remnant at any time provided that the circumburst medium has $n_{0} \lesssim 1 \mathrm{~cm}^{-3}$. This conclusion relies on three fortunate coincidences. First and most importantly, the shell formation time coincidentally satisfies $t_{\mathrm{sf}} \sim \tau_{\mathrm{Mw}}$ for $n_{0}=1 \mathrm{~cm}^{-3}$. Although some fraction of GRBs may occur in dense molecular clouds, recent modeling of jetted afterglows indicates that the ambient density is in the range $n_{0} \sim 10^{-3}$ to $10^{1.5} \mathrm{~cm}^{-3}$ (Berger et al. 2000, 2001; Harrison et al. 2001; Panaitescu \& Kumar 2001a). Second, in contrast to $e^{+}$created by $\mathrm{SNe}, \mathrm{GRB}$ positrons annihilate on a timescale $\tau_{\mathrm{ann}} \gtrsim t_{\mathrm{sf}}$. Third, $T_{\mathrm{ann}} \gtrsim 5 \times$ $10^{5} \mathrm{~K}$, so that a clear line from the direct annihilation channel is visible throughout the source lifetime.

Two backgrounds could contaminate the annihilation line signal. First, radioactive decay of heavy elements in SNe creates substantial numbers of positrons; however, we have argued in $\S 2$ that the number of positrons surviving the early stages of the $\mathrm{SN}$ is small. Numerical models of SNe (e.g., Chan \& Lingenfelter 1993) coupled with observations of positron annihilation radiation in the Milky Way (e.g., Purcell et al. 1997) imply that the number of $e^{+}$produced in a GRB exceeds the number produced in an SN provided that $\xi_{-2.3} \geq 0.002$. A second background is due to inverse Compton (IC) emission by 
the electrons accelerated at the collisionless shock bounding the remnant. The recent detection of $\mathrm{TeV}$ gamma-ray emission from SN remnants (Muraishi et al. 2000; Tanimori et al. 1998) coupled with the well-observed high-energy synchrotron emission from the same remnants (Koyama et al. 1995, 1997) implies an IC signal of $F_{\gamma, \text { IC }} \lesssim 10^{-12} d_{10}^{-2}\left(\epsilon_{\gamma} / 511 \mathrm{keV}\right)^{-1.5} \mathrm{~cm}^{-2} \mathrm{~s}^{-1}$ $\mathrm{keV}^{-1}$ (assuming the spectral indices of the radio synchrotron and IC radiation are identical; Reynolds 2001), much smaller than the annihilation signal. Relativistic protons accelerated by the shock can create positrons through the decay of $\pi^{+}$produced in collisions with thermal protons. However, using the formalism of Furlanetto \& Loeb (2002), we find that this production channel is negligible so long as $\xi_{-2.3} \geqslant 10^{-6}$.

Existing observations (Purcell et al. 1997; Kinzer et al. 1996, 2001) have shown an excess of positron annihilation radiation toward the Galactic center, but the angular resolution of the measurements is too poor to identify individual sources. Nevertheless, the prospects for detection of young GRB remnants in the Milky Way are good, provided that GRB sources are not confined to dense molecular clouds. As shown in Figure 1, a GRB remnant produces a characteristic annihilation line photon flux of $F_{\gamma} \sim 3 \times 10^{-5} \xi_{-2.3} n_{0} / d_{10}^{2} \mathrm{~cm}^{-2} \mathrm{~s}^{-1}$. The International Gamma-Ray Astrophysical Laboratory (INTEGRAL) satellite, ${ }^{2}$ expected to be launched in 2002 October, will have spectral capabilities in the energy range of interest. For a $10^{6} \mathrm{~s}$ observation, the Spectrometer on INTEGRAL and the Imager on Board the INTEGRAL Satellite (IBIS) have $3 \sigma$ line sensitivities of $\sim 5 \times 10^{-6}$ and $10^{-4} \mathrm{~cm}^{-2} \mathrm{~s}^{-1}$, respectively. The Energetic $X$ ray Imaging Survey Telescope (EXIST), ${ }^{3}$ a proposed all-sky hard $\mathrm{X}$-ray survey mission, has an expected $5 \sigma$ line sensitivity of $\sim 5 \times 10^{-6} \mathrm{~cm}^{-2} \mathrm{~s}^{-1}$ in the relevant energy range (assuming an integration time of $10^{7} \mathrm{~s}$, the mean exposure time planned for any point on the sky). These instruments will also have the spectral resolution to identify the line. The IBIS instrument on

\footnotetext{
${ }^{2}$ See http://astro.estec.esa.nl/SA-general/Projects/Integral/integral.html.

${ }^{3}$ See http://exist.gsfc.nasa.gov.
}

INTEGRAL and the EXIST survey, with angular resolutions of $12^{\prime}$ and 5', respectively, will also be able to map nearby GRB remnants, for which the angular size at $t_{\mathrm{sf}}$ is $\sim 14 E_{51}^{2 / 7} n_{0}^{-3 / 7} d_{10}^{-1}$ arcmin.

If GRBs occur primarily in dense environments, $t_{\mathrm{sf}}$ decreases and the probability for observing a remnant declines. Figure 1 shows that the average flux in the annihilation line from a nearby extragalactic source $(d \gtrsim 1 \mathrm{Mpc})$ during the Sedov phase will likely remain below the sensitivity limits of the above instruments. However, once shell formation begins, the positrons will annihilate through charge exchange with recombining hydrogen on a timescale $\tau_{\mathrm{cx}} \sim 10 /\left(f_{\mathrm{H}_{\mathrm{I}}} n_{\mathrm{sh}}\right) \mathrm{yr}$, where $f_{\mathrm{H}_{\mathrm{I}}}$ is the neutral fraction (Bussard et al. 1979). If the shell is supported primarily by magnetic and cosmic-ray pressure, Cox et al. (1999) find that the shell density at $t_{\mathrm{sf}}$ is $n_{\mathrm{sh}} \sim 100 B_{0}^{-1} n_{0}^{23 / 14} E_{51}^{1 / 14} \mathrm{~cm}^{-3}$, where $B_{0}$ is the transverse component of the ambient magnetic field in units of microgauss. The maximal annihilation flux is then $F_{\gamma, \mathrm{sh}} \sim 3 \times 10^{-3} f_{\mathrm{H} \text { I }} \xi_{-2.3} E_{51}^{15 / 14} n_{0}^{23 / 14} B_{0}^{-1} d_{\mathrm{Mpc}}^{-2} \mathrm{~cm}^{-2} \mathrm{~s}^{-1}$, where $d_{\mathrm{Mpc}}$ is the distance to the GRB in megaparsecs. Because the charge exchange process produces Ps, $75 \%$ of the annihilation energy is released in the continuum, but this suggests that the remnants will brighten significantly during the brief phase of shell formation and may be visible out to the Virgo Cluster distance, $d \sim 20 \mathrm{Mpc}$. Given the number of galaxies in Virgo, we would therefore expect to find at least one Virgo remnant in this stage provided that $f_{\mathrm{H}_{\mathrm{I}}} n_{\mathrm{sh}} \lesssim 1 \mathrm{~cm}^{-3}$. The details of the luminosity and spectrum will depend critically on modeling the process of shell formation and cooling. Such modeling stretches the limits of current simulations (e.g., Blondin et al. 1998; Cox et al. 1999) but may become feasible in the future.

We thank J. Raymond for helpful discussions. This work was supported in part by NASA grants NAG5-7039 and NAG57768 and NSF grants AST 99-00877 and AST 00-71019 for A. L.; S. R. F. acknowledges the support of an NSF graduate fellowship.

\section{REFERENCES}

Ayal, S., \& Piran, T. 2001, ApJ, 555, 23

Berger, E., et al. 2000, ApJ, 545, 56 2001, ApJ, 556, 556

Blondin, J. M., Wright, E. B., Borkowski, K. J., \& Reynolds, S. P. 1998, ApJ, 500,342

Bloom, J. S., et al. 1999, Nature, 401, 453

Bussard, R. W., Ramaty, R., \& Drachman, R. J. 1979, ApJ, 228, 928

Cavallo, G., \& Rees, M. J. 1978, MNRAS, 183, 359

Chan, K., \& Lingenfelter, R. E. 1993, ApJ, 405, 614

Chevalier, R. A., Blondin, J. M., \& Emmering, R. T. 1992, ApJ, 392, 118

Cioffi, D. F., McKee, C. F., \& Bertschinger, E. 1988, ApJ, 334, 252

Cox, D. P., et al. 1999, ApJ, 524, 179

Dermer, C. D., \& Böttcher, M. 2000, ApJ, 534, L155

Djorgovski, S. G., et al. 2001, preprint (astro-ph/0107535)

Frail, D. A., et al. 2001, ApJ, 562, L55

Freedman, D. L., \& Waxman, E. 2001, ApJ, 547, 922

Furlanetto, S. R., \& Loeb, A. 2002, ApJ, in press (astro-ph/0201313)

Gieseler, U. D. J., Jones, T. W., \& Kang, H. 2000, A\&A, 364, 911

Gould, R. J. 1989, ApJ, 344, 232

Harrison, F. A., et al. 2001, ApJ, 559, 123

Jun, B., Jones, T. W., \& Norman, M. L. 1996, ApJ, 468, L59

Kinzer, R. L., et al. 1996, A\&AS, 120, C317 2001, ApJ, 559, 282

Koyama, K., et al. 1995, Nature, 378, 255 1997, PASJ, 49, L7

Kulkarni, S. R., et al. 2000, Proc. SPIE, 4005, 9

Loeb, A., \& Perna, R. 1998, ApJ, 503, L35

Mészáros, P., Ramirez-Ruiz, E., \& Rees, M. J. 2001, ApJ, 554, 660

Milne, P. A., The, L.-S., \& Leising, M. D. 1999, ApJS, 124, 503
Muraishi, H., et al. 2000, A\&A, 354, L57

Ore, A., \& Powell, J. L. 1949, Phys. Rev., 75, 1696

Paczyński, B. 2001, Acta Astron., 51, 1

Panaitescu, A., \& Kumar, P. 2001a, ApJ, 554, 667

. 2001b, ApJ, 560, L49

Perna, R., Raymond, J., \& Loeb, A. 2000, ApJ, 533, 658

Piran, T. 2000, Phys. Rep., 333, 529

Piran, T., \& Ayal, S. 2002, in Disks of Galaxies: Kinematics, Dynamics, and Interactions, ed. E. Athanassoula \& A. Bosma (San Francisco: ASP), in press (astro-ph/0203003)

Piran, T., Kumar, P., Panaitescu, A., \& Piro, L. 2001, ApJ, 560, L167

Porciani, C., \& Madau, P. 2001, ApJ, 548, 522

Purcell, W. R., et al. 1997, ApJ, 491, 725

Reichart, D. E. 2001, ApJ, 554, 643

Reynolds, S. P. 2001, Space Sci. Rev., 99, 177

Rhoads, J. E. 1997, ApJ, 487, L1

Schmidt, M. 2001, ApJ, 552, 36

Sedov, L. I. 1959, Similarity and Dimensional Methods in Mechanics (New York: Academic)

Shemi, A., \& Piran, T. 1990, ApJ, 365, L55

Svensson, R. 1982, ApJ, 258, 321

Svensson, R., Larsson, S., \& Poutanen, J. 1996, A\&AS, 120, C587

Tanimori, T., et al. 1998, ApJ, 497, L25

Thompson, C., \& Madau, P. 2000, ApJ, 538, 105

Truelove, J. K., \& McKee, C. F. 1999, ApJS, 120, 299

van Paradijs, J., Kouveliotou, C., \& Wijers, R. A. M. J. 2000, ARA\&A, 38, 379

Woods, E., \& Loeb, A. 1999, preprint (astro-ph/9907110) 